\title{
Integrating Collective Work Aspects in the Design Process: An Analysis Case Study of the Robotic Surgery Using Communication as a Sign of Fundamental Change
}

\author{
Anne-Sophie Nyssen and Adelaide Blavier \\ University of Liege, Laboratory of Cognitive Ergonomics, \\ 5 boulevard du rectorat, B32, 4000 Liège, Belgium \\ asnyssenaulg.ac.be
}

\begin{abstract}
Ergonomic criteria are receiving increasing attention from designers but their applications don't ensure that technology matches the system's constraints and its reliability. The aim of this paper is to study how robotic surgery induces fundamental changes in the collective work using communication as a sign of the adaptation processes. First, we compared verbal communication between surgeons in two conditions (laparoscopy and robotic surgery). Secondly, we compared three teams with different level of expertise with the robotic system on a repeated surgery act in order to identify permanent and transitory changes. Third, we analyzed conversion cases. We showed more acts of communication with the robotic system. The content analyses of communication revealed a profound change of the structure of the task that requires explicit collaborative modes. Although our sample is small, our results can be extended in other domains concerned with telework.
\end{abstract}

Keywords: Robotics, collective work, adaptation processes, design, assessment.

\section{Introduction}

The number, complexity and variety of medical devices have increased in recent years. At the same time, human error is considered to be the major contributing factor of medical accidents. Accident investigations are traditionally based on epidemiological methods rather than on detailed analyses of work situations. These methods often classify accidents into exclusive categories: human error, equipment failure or unavoidable complication. We can ask ourselves if such a classification still makes sense in our modern world where human, techniques and organization are interdependent. The health care system is characterized by diversity, complexity and the need for coordinated work between multiple disciplines. This has caused great difficulty in the design of clinical technical systems. Designers can be some kind of dreamers; they discover how difficult it is to assist activity in naturalistic situations. Many technical aids are not used, are misused or induce new forms of errors. This paradox was depicted by Bainbridge [1] for automated systems as the irony of automation. Among the reasons for these failures we can quote [2]: 1) a large mismatch between aid support and users' real needs, 2) the communication gap between potential users and computer science, for example, the role of the aid is often unclear for

The original version of this chapter was revised: The copyright line was incorrect. This has been corrected. The Erratum to this chapter is available at DOI: 10.1007/978-3-642-11750-3_10

P. Palanque, J. Vanderdonckt, and M. Winckler (Eds.): HESSD 2009, LNCS 5962, pp. 18-27, 2010.

(C) Springer-Verlag Berlin Heidelberg 2010 
the user, 3) the absence of a coherent design philosophy: for instance, the method of knowledge representation may be inappropriate, 4) the disregard of organizational issues: the complex environment where the system is used is not taken into account, nor are its dynamics and uncertainty.

Regarding the unintended side effects of technology, several researchers have indicated the need to reevaluate the human-machine interaction at a fundamental level $[3,4,5,6]$. The concept of user-centered design refers to this attempt. The fundamental principles of such design approaches are: involvement of target-users in the design process, action-facilitation design and scenario-based design. Even if accepting the centrality of the user in the design process is becoming a more accepted prerequisite of appropriate person-machine design, its application has often been limited in practice to some particular design stages. A look at the design cycle schematized by Wickens, Gordon and Liu [7] illustrates the common practice of failing to involve the users. At the beginning of the cycle, potential users rarely converse with designers. It is the "human factors professionals", sometimes psychologists, sometimes ergonomists, who provide designers with the frame of reference concerning the task, the work environment and users' needs. As the prototype is developed, users are more easily included in the design process, especially for the validation of the prototype. At the end of the design process, the functionality of the product is assessed sometimes in real use, for a period of time. However, at this late stage, changing the product becomes unfeasible and procedures or training measures constitute, for the most part, the protective measures that ensure safety of the joint cognitive system.

Conducted in this way, none of the above stages relate specifically to a user in context centric view. The process places the product at the center.

From an activity theory perspective [8,9], aid systems should be designed to support operators in doing a task safely and efficiently in real work situations. Cognitive activity analysis as developed by Rasmussen and Vicente [10], is placed at the center of the analysis, focusing on information, mental effort, decision making and regulation. The concept of ecological interface was developed to illustrate an interface that provides appropriate support for the different levels of cognitive functioning.

Along the same line, but this time stressing the contextual and social point of view, is the Scenario-Based Design approach, a set of perspectives linked by a radical vision of user-oriented design [11]. This approach is not entirely new. For decades, systems developers have spontaneously used scenarios to envision future concrete use of their systems. But this informal practice has gained international acknowledgment, and the social content of the work is taken into account. To integrate context into the design, the task analysis stems from a scenario: "One key element in this perspective is the user-interaction scenario, a narrative description of what people do and experience as they try to make use of computer systems and applications. Computer systems and applications can and should be viewed as a transformation of user tasks and their supporting social practices" [11, pp 3]. Despite these valuable insights, scenarios constitute only examples of interactions of use and thus suffer from incompleteness.

We use one study to illustrate how important in-depth work analysis is in evaluating and designing new technology. More than 600 hours of observation were conducted in the operating rooms selected on the basis of their use of the new robotic system. 


\section{Robotic Surgery System}

Surgery has known important developments with technological advances. Laparoscopy is certainly one of them. There is little question that laparoscopy represents a definite progress in patient's treatment. However, there are a lot of drawbacks, some of which are not without significance. For instance, the surgeon has lost all tactile feedback, (s)he has to perform operation with only sensory input from the twodimensional picture on a video screen, and the procedure, to be done with long instruments, is seldom performed in a comfortable position for the surgeon. The fact that long instrument are used through an opening (trocar) in the abdominal wall, limits the degrees of freedom of the surgeon to a number of 4: in and out, rotation around the axis, up and down and from medial to lateral. The aim of the computer guided mechanical interface, commonly referred to as a robot, is to allow for 1) restoration of the degrees of freedom that were lost, thanks to an intra-abdominal articulation of the surgical tools, 2) three dimensional visualization of the operative field in the same direction as the working direction, 3) modulation of motion amplitude by stabilizing or by downscaling and 4) remote control surgery. Because of these improvements, the surgical tasks can be performed with greater accuracy.

However, to place a computer as an interface between the surgeon and the patient transforms the joint cognitive system.

Laparoscopy procedures typically involve the simultaneous use of three or more instruments (e.g. laparoscope, probe or gripper and shears or other cutting tools). Because of this, at least one tool must be operated by an assistant. The assistant's task is often limited to static functions of holding the instrument and managing the camera.

In classical laparoscopy, the assistant and the surgeon are face to face, and they use the same 2D representation of the surgical field to tailor the task.

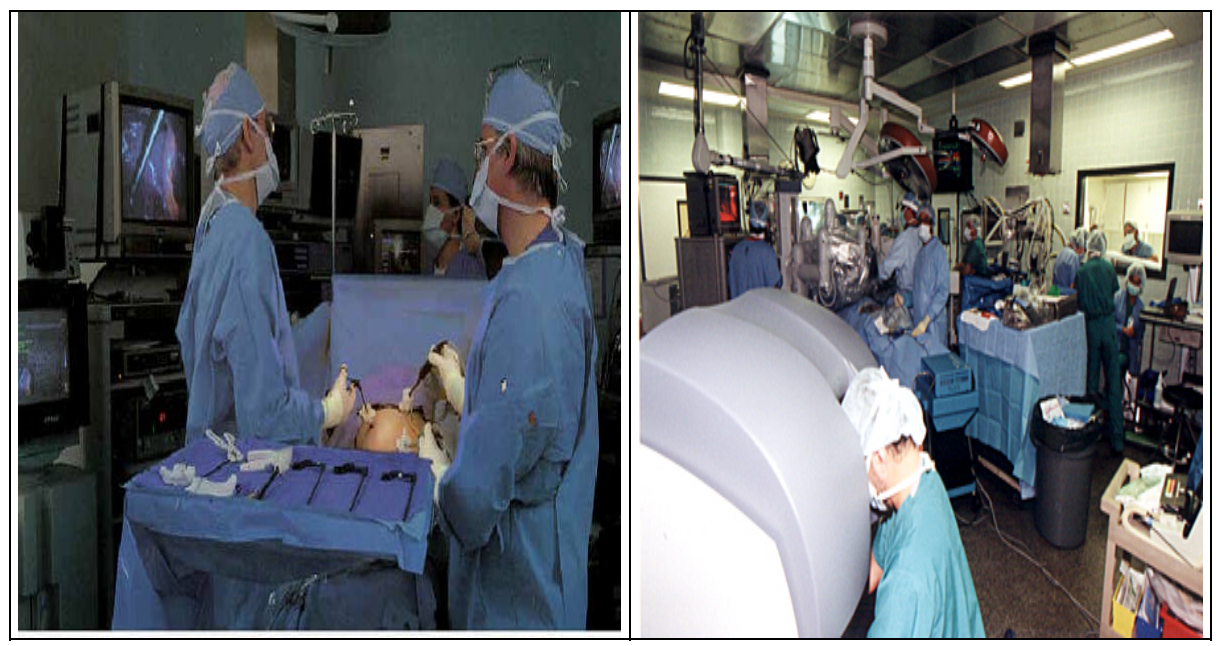

Fig. 1. Configuration of the operating theater in classical laparoscopy (left) and with the robotic system (right) 
In robotic surgery, the surgeon is seated in front of the console at a distant point, looking at an enlarged three-dimensional binocular display on the surgical field while manipulating handles that transmit the electronic signals to the computer that transfer the exact same motions to the robotic arms. Robotic surgery can be performed at distant locations. However, within the actual technological system, the surgeon is still in the same operating room as the patient. The computer-generated electrical impulses are transmitted by a 10 -meter long cable that controls the three articulated "robot" arms. Disposable laparoscopic articulated instruments are attached to the distal part of two of these arms. The third arm carries an endoscope with dual optical channels, one for each of the surgeon's eyes, which allows a true binocular depth perception (stereoscopy). The assistant is next to the patient, holding one or two instruments and looking at a 2-D display of the surgical field.

\section{Communication as a Sign of Adaptation Requirements}

Every act of communication, both verbal and non verbal, can be considered as an adaptive process analogous to biological evolution. Adaptation is the process of adjusting the mental structures and the behavior to cope with changes. Because so much of the adaptation processes in real time within the health care system are still verbal communication, the analysis of language becomes an important paradigm in order to study the adaptation capacities of a system facing a change.

When practitioners repeatedly work together, a reduction of verbal information exchanges is observed as practitioners get to know each other. Information taken directly from the work field replaces the verbal exchanges. Indeed, any regular action, parameter or alarm takes on the character of the "initiator" of verbal communication $(12 ; 13 ; 14)$. Other studies (i.e. 15) have examined the relationship between communication and non routine situations in complex systems: the greater the trouble, the greater are the demands for information centered on the task across the members of the team.

Based on the above arguments, three important points can be noted. First, the environment provides feedback, which is the raw material for adaptation. Simple systems tend to have very straightforward feedback, where it often easy and instantaneous to see the result of an action. Complex systems may have less adequate feedback. The deployment of technology has increased the complexity of communication from non verbal to verbal, and to complex symbolic patterns. Additionally, introducing media and a distance between the agent and the process to control can delay and/or result in loosing feedback information. In laparoscopy surgery, the surgeon looses direct contact with the surgical site. S/he looses tactile feedback and performs operations with only sensory input from the video picture. As the robotic system is introduced in the $\mathrm{OR}, \mathrm{s} / \mathrm{he}$ looses proprioceptive feedback in addition to loosing a face to face feedback communication channel.

Secondly, communication is a dynamic feedback process which, in turn, affects the communicators. As we shall see, because the assistant and the surgeon have often prior knowledge and experience with the task, the assistant can anticipate the next movement or instrument that the surgeon needs in a routine task and non verbal communication can be very efficient (e.g., when the surgeon makes a hand signal to 
indicate to stop the movement or when s/he looks at the assistant to verify the receipt of an implicit request).

Third, in this dynamic perspective, short term adaptation feedback strategies that are exclusively based on verbal communication can be highly resource-consuming for the practitioners over time and, thus, may lead to long term inadequate adaptation.

Each of these points will be dealt with in our working hypotheses.

- In the case of adaptation, it is hypothesized that the technical system provides good feedback that supports the system to carry the task. Within our framework that views communication as an adaptive process, the following can be expected with the introduction of a robot system:

o in the short term, new patterns of communication that reveal adaptation strategies

○ with training and regular interactions, a reduction of communication that reveals the dynamic nature of the adaptation process

- In the case of lack of or inappropriate adaptation, the technical system provides inadequate feedback resulting in increasing and maintaining the verbal communication to compensate for the weakness of feedback from the new equipment.

\section{Experimental Study and Verbal Communication Analysis}

We carried out three studies to examine our hypotheses:

1. First, we compared surgical operations that were performed with a robotic system compared with classical laparoscopy. We chose two types of surgery procedures (digestive and urology) because it is possible to perform them with either classical laparoscopy or with a robotic system.

In the two conditions (robotic and classical laparoscopy), the team members were identical. They were experts in the use of classical laparoscopy ( $>100$ operations) and were at least familiar with the use of the robotic system ( $>10$ operations). We observed 5 cholecystectomy (digestive) with the robotic system and 4 with classical laparoscopy, and 7 prostatectomy (urology) with the robotic system and 4 with classical laparoscopy.

The robotic system used in our study was the Da Vinci robotic system (Intuitive Surgical, Mountain View, CE, USA) as shown in Figure1.

2. Secondly, we compared teams with different levels of expertise with the robotic system during gynecology surgery. We compared three teams with different levels of expertise who successively performed two tubular reanastomosis of 36 Fallopian tubes: 1) both the surgeon and the assistant were experts with the robotic system $(>50$ operations with the robotic system), 2) the surgeon was an expert while the assistant was a novice with the robotic system ( $<10$ operations with the robotic system), 3 ) the surgeon and the assistant were novices with the robotic system ( $<10$ operations with the robotic system).

3. Thirdly, we compared routine and non routine operations: conversion from robot surgery to classical surgery.

In the three studies, we recorded all the verbal communication between the surgeon and the assistant. We analyzed their content and identified six categories of 
communication. We also measured the duration of the intervention, as this is an important performance criterion for surgeons.

The six types of communication were:

- Verbal demands concerning the orientation (and localization) of organs.

- Verbal demands concerning the manipulation of instruments and/or organs.

- Explicit clarification concerning strategies, plans and procedures.

- Orders referring to tasks such as cutting, changing instruments, and cleaning the camera.

- Explicit confirmation of detection or action.

- Other communications referring to state of stress or relaxation.

For each category, we measured the number of acts of communication, while taking into account the duration of the surgery (ratio = number of acts of communication / time (in seconds) $X$ 100). The Mann-Whitney $U$ test was used to compare the two techniques: classical laparoscopy and robotic surgery and the Kruskal-Wallis test was used across the board.

\section{Results}

\subsection{Communication as a Feedback Adaptive Process}

The average duration of the intervention was significantly longer $(\mathrm{p}<0.05)$ with the robotic system (cholecystectomy: $82.59 \pm 27.37$; prostatectomy: 221.39 \pm 58.79 ) than with

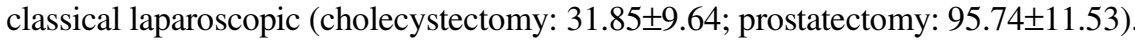

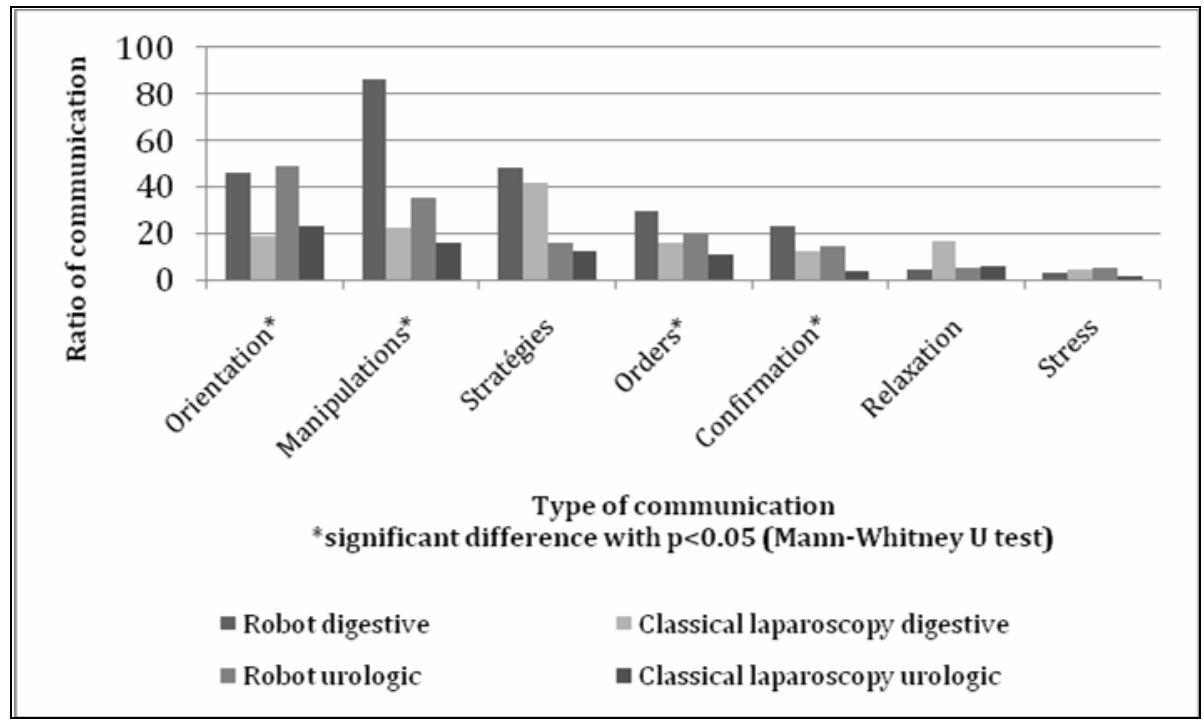

Fig. 2. Communications during robotic and classical laparoscopy in digestive and urologic surgery 
Figure 2 shows that the introduction of the robotic system created a new pattern of communication. This pattern of communication was similar for the two types of surgery.

The significant increase in the number of communication acts $(p<0.05)$ referring to orientation, manipulation, order and confirmation within the robot system suggests that a breakdown occurs in the collaboration between the surgeon and the assistant. The surgeon works alone and continually needs to ask the assistant about the orientation and the placement of the instrument (which is manipulated by the assistant) in order to facilitate the identification of the organs as demonstrated in the following example of interaction:

Surgeon at the consol: "could you tell me if you are touching something here, because I see a particularity"

Assistant surgeon near the patient: "yes, I am touching something hard - it is a bone".

Explicit demands, order, and confirmation are needed because the system configuration impedes face to face implicit control and anticipation of the actions.

\subsection{Communication as a Dynamic Adaptation Processes: Permanent and Transitory Changes}

Our experimental plan allows us to identify the permanent and the transitory changes induced by the change of equipment.

Our results show that the number of acts of communication is reduced with repeated experience: from the first operation to the second operation of Fallopian tube anastomosis, but also with the degree of expertise of the team with the robotic system (see fig. 3).

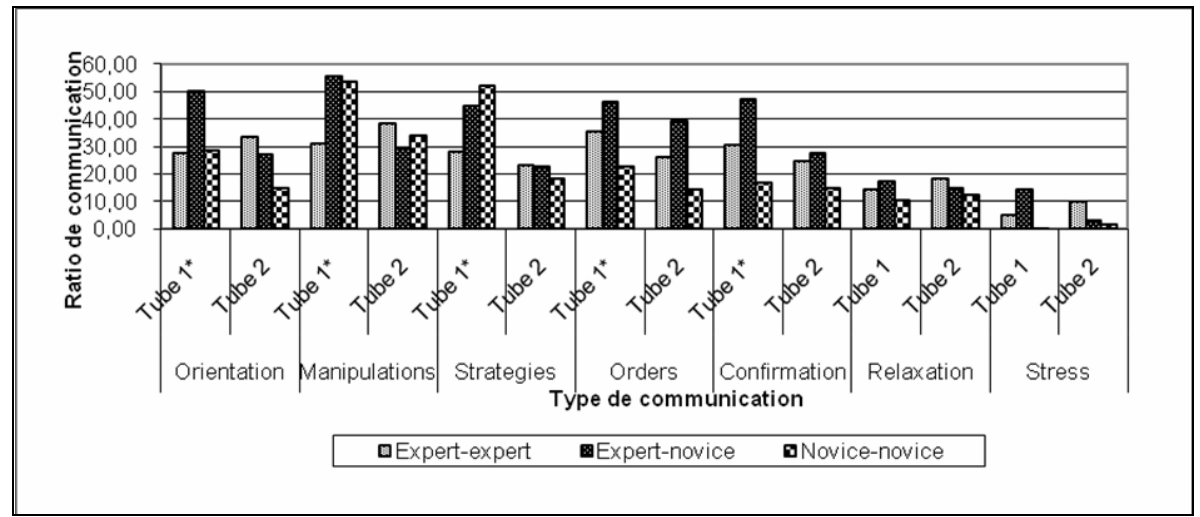

Fig. 3. Communication during first and second tube anastomosis according to the expertise

Detailed analysis of communication showed that the number of communication acts referring to orientation, manipulation and strategies was significantly reduced $(\mathrm{p}<0.05)$ when both surgeons were experts in robotic surgery and from the $1^{\text {st }}$ tube to the $2^{\text {nd }}$ tube. Not surprisingly, the number of acts of communication referring to order and confirmation was significantly greater when an expert was present in the team. 
We observed that this increase of order and confirmation does not change from the $1^{\text {st }}$ tube to the $2^{\text {nd }}$ tube and is maintained within the experts' team.

\subsection{Communication as a Sign of Trouble}

We observed two conversions: 1 in urology from a robotic surgery to open surgery and 1 in digestive surgery from robotic surgery to classical laparoscopy surgery.

Each of these conversions is associated with an increased number of verbal communications (see fig.4). These communications concerned explicit clarification of strategies (replanning) and expectations concerning orientation and manipulations. We also observed less communication that referred to confirmation. During a crisis, the surgeon seems to act; he does not take the time to verify the receipt of his action or request.

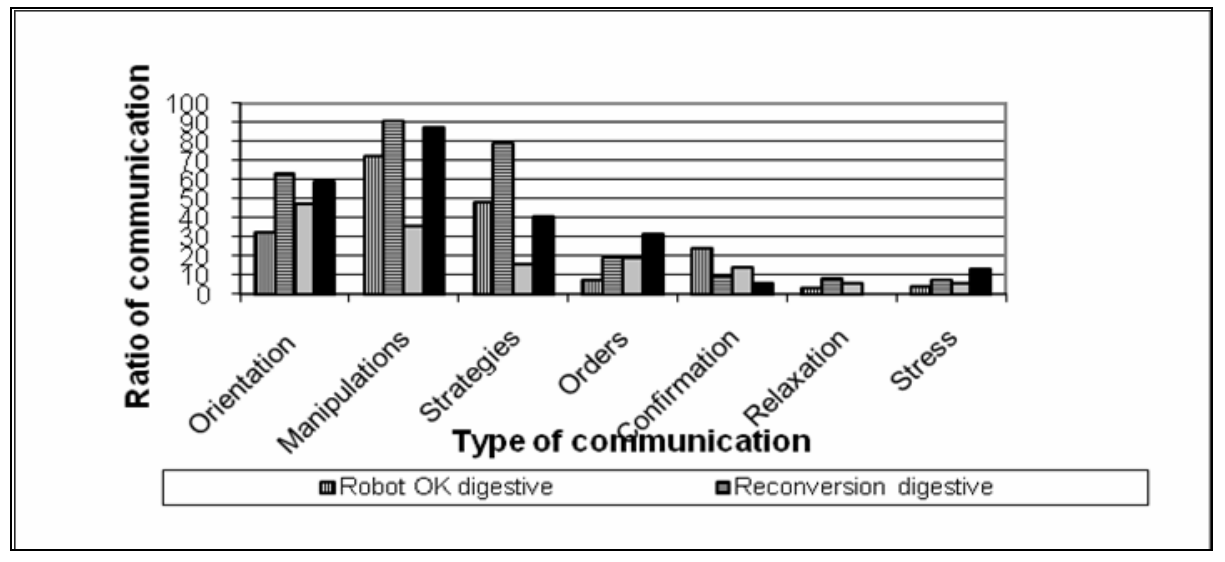

Fig. 4. Communication during first and second tube anastomosis according to the expertise

\section{Discussion}

Based on our results, it is clear that the robotic system changes the feedback loop and that verbal communication used by surgeons is a feedback-adaptive process to compensate the feedback information absent in the robotic system. Our results show that both the number of communication acts and the type of communication evolves with the agent-robot environment interactions, suggesting some kind of successful adaptation to the change of equipment. It seems that manipulation, orientation and strategies can be rapidly learned through interaction with the technical system (from tube 1 to tube 2.). However, orders and confirmation are maintained within the experts' team. This result suggests that, by introducing a distance between the surgeon and the assistant, the robotic system changes profoundly the structure of the task and the mode of cooperation between the surgeon and his assistant. It favors an explicit division of work and an explicit leadership based on order and continual control of the work (confirmation). As a result, the status of the assistant and of the OR team are modified. The surgeon assistant becomes more like a technician, responding to the orders 
of the surgeon. There are two new actors in the team: the robot and the robot technician who become essentials. We can predict an impact of these changes on the OR team work satisfaction associated with new forms of errors such as a loss of "situational awareness".

As mentioned earlier, when complications occur, increased verbal communication is required to clarify plans and expectations in order to enable rapid coordinated actions between the surgeon and the assistant and to maintain a update shared situational awareness. These conversion cases show how the surgeons, and not the robot, have mechanisms for recovering from the situation before it affects the patient by replanning the cases into classical surgery. This means that the system's capacity for facing unexpected events resides in the human part rather than in the technical part of the system. Indeed, adaptation emerges through the history of different agentenvironment coupling over time (open surgery, classical laparoscopic surgery, robotic surgery) that enhances the agent's autonomy towards the variability from the environment (for eg. a technical change).

Although recent work from Joint Cognitive systems engineering [16] discusses issues like autonomy, variability and resilience, much prevention effort is still spent on automation and standardization. Our results captured the idea that studying both the behavior of the system facing a change provides markers on the system's adaptation capacity and, in turn, will help to develop technology that enhances better adaptative coupling between agents and their changing environment.

\section{Concluding Remarks}

Ergonomic criteria are receiving increasing attention from designers but their application doesn't ensure that technology matches the system's constraints and enhances its reliability. Although we cannot predict the future, we may attempt to better guide the design process by adopting a systemic view. Our aim is to insist to assess the impact of technology changes on all the dimensions of a work situation: technical, economic, performance, cognitive, and organizational. In the health care system, as in other complex and dynamic systems, there is a need for researchers and designers to more investigate the impact of the equipment on the reciprocal interaction between cognition and organization. Doing so is critical for the quality, safety and effectiveness of the modern work.

\section{References}

1. Bainbridge, L.: The ironies of automation. In: Rasmussen, J., Duncan, K., Leplat, J. (eds.) New technology and human error, pp. 271-283. Wiley, London (1987)

2. O'Moore, R.: The conception of a medical computer system. In: van Gennip, E.M.S.J., Talmon, J.L. (eds.) Assessment and evaluation of information technologies in medicine, pp. 45-49. IOS Press, Amsterdam (1985)

3. Sheridan, T.B.: Human centered automation: Oxymoron or common sense? presented at the industrial summer school on human-centered automation, Saint-Lary, France (1995)

4. Norman, D.A.: The invisible computer. MIT Press, Cambridge (1999)

5. Norman, D.A.: Things that make us smart: Defending human attributes in the age of the machine. Addison-Wesley, Reading (1993) 
6. Billings, C.E.: Aviation automation: The search of a human-centered approach. Lawrence Erlbaum Associates, Mahwah (1997)

7. Wickens, C.D., Gordon, S.E., Liu, Y.: An introduction to human factors engineering. Longman, New York (1997)

8. Roe, R.A.: Acting systems-design - an action theoretical approach to the design of mancomputer systems. In: De Keyser, V., Qvale, T., Wilpert, B., Ruiz Quintanilla, S.A. (eds.) The meaning of work and technical options, pp. 179-195. Wiley, Chichester (1988)

9. Hacker, W.: Activity: A fruitful concept in industrial psychology. In: Frese, M., Sabini, J. (eds.) Goal directed behavior: The concept of action in psychology, pp. 262-383. Lawrence Erlbaum Associates, Hillsdale (1985)

10. Rasmussen, J., Vicente, K.L.: Coping with human errors through system design: implications for ecological interface design. International Journal of Man-Machine Studies 31, 517-534 (1989)

11. Caroll, J.M.: Scenario-based design. Envisioning work and technology in system development. Wiley, New York (1997)

12. Savoyant, A., Leplat, J.: Statut et fonction des communications dans l'activité des équipes de travail (Statut and function of the communications in the activities of the workteams). Psychol.fr. 28(3), 247-253 (1983)

13. Pavard, B.: Système coopératifs: de la modélisation à la coopération. Octares, Toulouse (1994)

14. Nyssen, A.S., Javaux, D.: Analysis of synchronization constraints and associated errors in collective work environments. Ergonomics 39, 1249-1264 (1996)

15. Bressolle, M.C., Decortis, F., Pavard, B., Salembier, P.: Traitement cognitif et organisationnel des micro-incidents dans le domaine du contrôle aérien: analyse des boucles de régulation formelles et informelles. In: De Terssac, G., Friedberg, E. (eds.) Coopération et conception, Octares, Toulouse, pp. 267-288 (1996)

16. Woods, D.D., Hollnagel, E.: Joint Cognitive Systems: Patterns in cognitive systems engineering. Taylor \& Francis Group, Boca Raton (2006) 